\title{
Instantaneous Transfer Entropy for the Study of Cardiovascular and Cardio-Respiratory Nonstationary Dynamics
}

\author{
Gaetano Valenza*, Member, IEEE, Luca Faes, Member, IEEE, Luca Citi, Member, IEEE, \\ Michele Orini, Member, IEEE, and Riccardo Barbieri, Senior Member, IEEE
}

\begin{abstract}
Objective: Measures of Transfer Entropy (TE) quantify the direction and strength of coupling between two complex systems. Standard approaches assume stationarity of the observations, and therefore are unable to track time-varying changes in nonlinear information transfer with high temporal resolution. In this study, we aim to define and validate novel instantaneous measures of transfer entropy to provide an improved assessment of complex non-stationary cardio-respiratory interactions.

Methods: We here propose a novel Instantaneous point-process Transfer Entropy $(i p T E)$ and validate its assessment as applied to cardiovascular and cardio-respiratory dynamics. In particular, heartbeat and respiratory dynamics are characterized through discrete time series, and modeled with probability density functions predicting the time of the next physiological event as a function of the past history. Likewise, non-stationary interactions between heartbeat and blood pressure dynamics are characterized as well. Furthermore, we propose a new measure of information transfer, the instantaneous point-process Information Transfer $(i p I n f T r$ ), which is directly derived from point-processbased definitions of the Kolmogorov-Smirnov distance.

Results and Conclusion: Analysis on synthetic data, as well as on experimental data gathered from healthy subjects undergoing postural changes confirms that $i p T E$, as well as $i p I n f T r$ measures are able to dynamically track changes in physiological systems coupling.

Significance: This novel approach opens new avenues in the study of hidden, transient, non-stationary physiological states involving multivariate autonomic dynamics in cardiovascular health and disease. The proposed method can also be tailored for the study of complex multi-system physiology (e.g., brain-heart or, more in general, brain-body interactions).
\end{abstract}

Index Terms-Transfer Entropy, Point Process, Heart Rate Variability, Complexity, Baroreflex, Respiratory Sinus Arrhythmia, Kolmogorov-Smirnov Distance

* Corresponding Author: g.valenza@ieee.org.

The research leading to these results has received partial funding from the Department of Anesthesia, Critical Care \& Pain Medicine, Massachusetts General Hospital, and Harvard Medical School, Boston, MA, USA.

G. Valenza is with the Computational Physiology \& Biomedical Instruments group, Bioengineering and Robotics Research Center E. Piaggio, and also with the Department of Information Engineering, University of Pisa, Italy.

L. Faes is with the IRCS-FBK and BIOtech, Dept. of Industrial Engineering, University of Trento, Italy.

L. Citi is with the School of Computer Science and Electronic Engineering, University of Essex, Colchester, UK.

M. Orini is with the Institute of Cardiovascular Science, University College London, London, UK.

R. Barbieri is with the Department of Electronics, Informatics and Bioengineering, Politecnico di Milano, Milano, Italy.

G. Valenza and R. Barbieri are also with the Massachusetts General Hospital/Harvard Medical School, Boston, MA, USA.

\section{INTRODUCTION}

Cardiovascular structure and functions, including vascular anatomy, electrical conduction, heart rate and blood-pressure variability, as well as cardio-respiratory dynamics, are associated with complex spatial and temporal patterns that can be quantified through methodological approaches derived from the theory of complex dynamical systems [1]-[4]. These approaches go beyond standard time and frequency domain analyses, as they account for the nonlinear relationship between the magnitude of physiological system responses and the strength/amplitude of the system input [5]-[9].

To this extent, measures of entropy have been widely used to quantify the randomness and regularity of a physiological system given the analysis of time series originated by it [10] [12]. More specifically, during the last decades, application of entropy measures to heart rate variability (HRV) series has been proven very effective in characterizing healthy and pathological states involving cardiovascular control [4], [9], [13]-[25]. Heartbeat dynamics and its spontaneous fluctuations result from complex interactions between the sympathetic and parasympathetic (vagal) limbs of the autonomic nervous system (ANS) [26], as well as from multiple self-regulating, adaptive biochemical processes [26].

A significant contribution to heartbeat complex oscillations is given by a dynamical, mutual interplay with numerous other physiological subsystems (e.g., endocrine, neural, and respiratory) [13]-[15]. Main phenomena refer to Respiratory sinus arrhythmia (RSA), i.e., the modulation of HR due to respiratory drive to cardiac vagal motor neurons, and the baroreflex, i.e., changes of heart rate due to blood pressure and related cardiovascular mechanics [3], [27], [28]. In this context, transfer entropy (TE) [29] is a mathematical construct devised to measure the nonlinear directional amount of information transfer from one physiological variable to the other.

In the frame of cardiovascular research, TE measures have been successfully applied for assessing the baroreflex functions [30], [31], aging-related changes [32], and brainheart interactions [33] (see also [34]-[41] for methodological variants and references therein).

Nevertheless, all TE-related estimates proposed so far are unable to finely track the non-stationary information transfer from one system to another, with a high-resolution in time. A limitation of TE is that its estimation requires the data to be stationary within a short-time window. Furthermore, the 
intrinsic unevenly sampled nature of heartbeat events is often neglected, thus leading to the application of preliminary interpolation procedures that could affect complexity measures.

In this study, we overcome these limitations by proposing a new definition of TE having time-varying properties, and no need of interpolation techniques on the original physiological time series. The new definition relies on the theory of probabilistic point-processes applied to cardiovascular dynamics [42], [43]. Briefly, given a series of RR intervals, it is possible to estimate PDFs describing and predicting each heartbeat event considering short-time recordings. For physiological and computational reasons, a good choice for these PDFs is represented by Inverse-Gaussian distributions whose first-order moment is modeled through autoregressive functions of the past samples (i.e., past heartbeat events) [42], [43]. The pointprocess model of heartbeat dynamics automatically accounts for the unevenly spaced heartbeat intervals and allows the use of goodness-of-fit tools [42], [43]. Once all PDFs of the $\mathrm{RR}$ interval series are estimated, since they are defined in the continuous time, it is possible to elaborate the conditional PDF of the present RR given its past history, and the conditional PDF of the present RR given both its past and the past of respiratory or blood pressure dynamics. Thus, following classical TE definition, it is possible to obtain instantaneous transfer entropy estimates defined by the instantaneous point process Transfer Entropy measures, ipTE $(t)$. Additionally, we here propose a novel instantaneous measure of information transfer, namely the instantaneous point-process Information Transfer, $i p \operatorname{Inf} \operatorname{Tr}(t)$ which is directly derived from KolmogorovSmirnov distance calculations between conditional PDFs.

After validation using synthetic data, we show exemplary estimates of $i p T E$, as well as of $i p I n f T r$, using experimental data gathered from healthy subjects undergoing postural changes. Particularly, we consider the instantaneous quantification of the information transfer from the respiration to the heart rate, indicated with $i p T E_{R P \rightarrow R R}$ and $i p I n f \operatorname{Tr}_{R P \rightarrow R R}$, and from the blood pressure to the heart rate, indicated with $i p T E_{B P \rightarrow R R}$ and $i p \operatorname{Inf} \operatorname{Tr}_{B P \rightarrow R R}$. Of note, in order to compare our results with an instantaneous measure of self-entropy quantifying the self-information storage, we also show results from a recently defined complexity index, the inhomogeneous point-process approximate entropy ipApEn [9], which is defined through monovariate analysis of heartbeat dynamics data, and is also embedded within a point process framework.

\section{Materials And Methods}

The proposed instantaneous measures of information transfer take inspiration from the standard non-parametric definition of transfer entropy which, as mentioned above, follows the Granger's general principle of measuring the information that the past of the driver brings to the present of the destination above and beyond the information that is brought by its own past. In fact, while modeling a RR interval series, Granger causality aims to quantify the prediction error of a monovariate autoregressive model and a bivariate model which includes, e.g., respiratory dynamics.
Within a point-process framework, we have InverseGaussian PDFs describing and predicting each heartbeat event. These PDFs can indeed be parametrized through a linear combination of past heartbeat events (monovariate autoregressive model) or through a linear combination of past heartbeat events and a linear combination of past respiratory events (bivariate autoregressive model). Then, the proposed instantaneous transfer entropy measure $i p T E$ is directly derived from the TE classical definition in terms of conditional PDFs, whereas the proposed instantaneous measure of information transfer ipInf $T r$ refers to the instantaneous estimation of the Kolmogorov-Smirnov distances between PDFs from these mono- and bivariate models.

Mathematical and algorithmic details follow below, focusing on the specific derivation of instantaneous information transfer from respiration (RP events) and heart rate (RR events) through $i p T E_{R P \rightarrow R R}$ and $i p I n f \operatorname{Tr}_{R P \rightarrow R R}$. A similar procedure yields the instantaneous information transfer from blood pressure (BP events) and heart rate (RR events), $i p T E_{B P \rightarrow R R}$ and $i p I n f \operatorname{Tr}_{B P \rightarrow R R}$, whose derivation is omitted for brevity.

\section{A. Point-Process Models of Heartbeat Dynamics}

Given the R-wave events $\left\{u_{j}\right\}_{j=1}^{J}$ detected from the electrocardiogram, and $\mathrm{RR}_{j}=u_{j}-u_{j-1}>0$ as the $j^{\text {th }} \mathrm{R}-\mathrm{R}$ interval, the generic probability distribution of the waiting time $t-u_{j}$ until the next R-wave event given the information available at time $t^{\prime}$ is modeled as an Inverse-Gaussian model [42]:

$$
\begin{aligned}
& f\left(t \mid \mathcal{H}_{t^{\prime}}, \xi\left(t^{\prime}\right)\right)=\left[\frac{\xi_{0}\left(t^{\prime}\right)}{2 \pi\left(t-u_{j}\right)^{3}}\right]^{\frac{1}{2}} \\
& \quad \times \exp \left\{-\frac{1}{2} \frac{\xi_{0}\left(t^{\prime}\right)\left[t-u_{j}-\mu\left(t^{\prime}, \mathcal{H}_{t^{\prime}}, \xi\left(t^{\prime}\right)\right)\right]^{2}}{\mu\left(t^{\prime}, \mathcal{H}_{t^{\prime}}, \xi\left(t^{\prime}\right)\right)^{2}\left(t-u_{j}\right)}\right\},
\end{aligned}
$$

for $t>u_{j}$. The associated cumulative distribution function is defined as:

$$
F\left(t \mid \mathcal{H}_{t^{\prime}}, \xi\left(t^{\prime}\right)\right)=\int_{u_{j}}^{t} f\left(\tau \mid \mathcal{H}_{t^{\prime}}, \xi\left(t^{\prime}\right)\right) d \tau .
$$

For $t \in(0, T]$, and $0 \leq u_{1}<\cdots<u_{k}<u_{k+1}<$ $\cdots<u_{K} \leq T$ the times of the events, it is possible to define $N(t)=\max \left\{k: u_{k} \leq t\right\}$ as the sample path of the associated counting process. Its differential, $d N(t)$, denotes a continuous-time indicator function, where $d N(t)=1$ when there is an event, or $d N(t)=0$ otherwise. The left continuous sample path is defined as $\widetilde{N}(t)=N\left(t^{-}\right)=\lim _{\tau \rightarrow t^{-}} N(\tau)=$ $\max \left\{k: u_{k}<t\right\}=j$.

Assuming history dependence, the instantaneous first-order moment (mean) $\mu_{\mathrm{RR}}$ of the distribution $f^{a}\left(t \mid \mathcal{H}_{t}^{a}, \xi^{a}(t)\right)$ can be defined as:

- A monovariate, discrete-time, linear autoregressive system:

$$
\mu_{\mathrm{RR}}\left(t, \mathcal{H}_{t}^{a}, \xi^{a}(t)\right)=\gamma_{0}+\sum_{i=1}^{p} \gamma_{1}(i, t) \mathrm{RR}_{\widetilde{N}(t)-i}
$$

where $\mathcal{H}_{t}^{a}=\left(u_{j}, \mathrm{RR}_{j}, \mathrm{RR}_{j-1}, \ldots, \mathrm{RR}_{j-p+1}\right)$ is the history of the past heartbeat events, $\xi^{a}(t)=$ $\left[\xi_{0}^{a}(t), \gamma_{0}(t), \gamma_{1}(1, t), \ldots, \gamma_{1}(p, t)\right]$ is the vector of the 
time-varying parameters, and $\xi_{0}^{a}(t)>0$ is the shape parameter of the Inverse-Gaussian distribution.

Likewise, assuming history dependence, the instantaneous first-order moment (mean) $\mu_{\mathrm{RP}-\mathrm{RR}}$ of the distribution $f^{b}\left(t \mid \mathcal{H}_{t}^{b}, \xi^{b}(t)\right)$ can be defined as:

- A bivariate, discrete-time, linear system including heartbeat and respiratory dynamics.

$$
\begin{aligned}
\mu_{\mathrm{RP}-\mathrm{RR}}\left(t, \mathcal{H}_{t}^{b}, \xi^{b}(t)\right)= & \phi_{0}+\sum_{i=1}^{p} \phi_{1}(i, t) \mathrm{RR}_{\widetilde{N}(t)-i}+ \\
& +\sum_{j=1}^{q} \phi_{2}(i, t) \mathrm{RP}_{\widetilde{N}(t)-q} \text { (4) }
\end{aligned}
$$

with RP values are values from respiration dynamics sampled at R-wave times, $\mathcal{H}_{t}^{b}=$ $\left(u_{j}, \mathrm{RR}_{i}, \mathrm{RR}_{i-1}, \ldots, \mathrm{RR}_{i-p+1}, \mathrm{RP}_{j}, \mathrm{RP}_{j-1}, \ldots, \mathrm{RP}_{j-q+1}\right)$ the history of the past heartbeat and respiratory events, $\xi^{b}(t)$ = $\left[\xi_{0}^{b}(t), \phi_{0}(t), \phi_{1}(1, t), \ldots, \phi_{1}(p, t), \phi_{2}(1, t), \ldots, \phi_{2}(q, t)\right]$ the vector of the time-varying parameters and $\xi_{0}^{a}(t)>0$ and $\xi_{0}^{b}(t)>0$ the shape parameters of the InverseGaussian distribution.

\section{B. Parameter Estimation, Model Selection, Goodness-of-Fit}

The parameter vectors $\xi^{a}(t)$ and $\xi^{b}(t)$ are estimated using the Newton-Raphson procedure to maximize the local likelihood [43]. Because there is significant overlap between adjacent local likelihood intervals, the Newton-Raphson procedure starts at time $t$ with the previous local maximum-likelihood estimate at time $t-\Delta$, with $\Delta=0.005 \mathrm{~s}$. The time-varying estimation of $i p T E$ and $i p I n f T r$ starts from window length $W=90 \mathrm{~s}$, thus proving an instantaneous complexity assessment right after few seconds of observations [9]. We determine the optimal orders $\{p, q\}$ based on the model goodness-offit tools [42], which are based on the Kolmogorov-Smirnov (KS) test and associated KS statistics [42]. Autocorrelation plots are also considered to test the independence of the model-transformed intervals [42]. Once the order $\{p, q\}$ is determined, the initial model coefficients are estimated by the method of least squares [42]. The recursive, causal nature of the estimation allows to predict each new observation, given the previous history, independently at each iteration. The model and all its parameters are therefore also updated at each iteration without priors.

\section{Definition of Instantaneous Point-process Transfer Entropy}

The proposed instantaneous point-process transfer entropy $i p T E(t)$ has its foundation in the theoretical definition of transfer entropy, which in its standard form can be considered as a non-parametric nonlinear extension of Granger causality [44]. Considering the classical definition:

$$
T E_{X \rightarrow Y}(t)=\mathbb{E}\left[\log \frac{f_{Y_{t} \mid Y_{t}^{-}, X_{t}^{-}}\left(y_{t} \mid y_{t}^{-}, x_{t}^{-}\right)}{f_{Y_{t} \mid Y_{t}^{-}}\left(y_{t} \mid y_{t}^{-}\right)}\right]
$$

where $X_{t}^{-}$and $Y_{t}^{-}$denote the past history of the processes $X$ and $Y$, respectively, and $f_{Y_{t} \mid Y_{t}^{-}, X_{t}^{-}}\left(y_{t} \mid y_{t}^{-}, x_{t}^{-}\right)$ and $f_{Y_{t} \mid Y_{t}^{-}}\left(y_{t} \mid y_{t}^{-}\right)$are the conditional PDFs. We conceptually map $f_{Y_{t} \mid Y_{t}^{-}}\left(y_{t} \mid y_{t}^{-}\right)$with $f^{a}\left(t \mid \mathcal{H}_{t}^{a}, \xi^{a}(t)\right)$, and $f_{Y_{t} \mid Y_{t}^{-}, X_{t}^{-}}\left(y_{t} \mid y_{t}^{-}, x_{t}^{-}\right)$with $f^{b}\left(t \mid \mathcal{H}_{t}^{b}, \xi^{b}(t)\right)$. Then, we obtain:

$$
i p T E_{R P \rightarrow R R}(t)=\mathbb{E}\left[\log \frac{f^{b}\left(t \mid \mathcal{H}_{t}^{b}, \xi^{b}(t)\right)}{f^{a}\left(t \mid \mathcal{H}_{t}^{a}, \xi^{a}(t)\right)}\right]
$$

which, for Inverse-Gaussian PDFs, considering the equivalence with Kullback-Leibler (KL) divergence, can be derived in a closed form as follows (see full derivation in Appendix):

$$
\begin{aligned}
& i p T E_{R P \rightarrow R R}(t)=\frac{1}{2}\left[\ln \frac{\xi_{0}^{R R}}{\xi_{0}^{R P-R R}+}\right. \\
&\left.+\frac{\xi_{0}^{R P-R R}}{\xi_{0}^{R R}}-1+\frac{\xi_{0}^{R P-R R}\left(\mu_{R R}-\mu_{R P-R R}\right)^{2}}{\mu_{R P-R R}^{2} \mu_{R R}}\right]
\end{aligned}
$$

\section{Definition of Instantaneous measures of Information Trans- fer}

The KL-divergence can be interpreted as a measure of statistical distance. A natural extension to the theory presented above is to consider other measures. A statistical distance which is particularly relevant in the case of point processes is the KS-distance, defining a new information transfer measure ipInfTr as follows:

$$
\begin{aligned}
& i p \operatorname{Inf} \operatorname{Tr}(t)= \\
& \qquad k \max _{\tau>t}\left|F^{a}\left(\tau \mid \mathcal{H}_{t}^{a}, \xi^{a}(t)\right)-F^{b}\left(\tau \mid \mathcal{H}_{t}^{b}, \xi^{b}(t)\right)\right| .
\end{aligned}
$$

Given $f^{a}\left(t \mid \mathcal{H}_{t}^{a}, \xi^{a}(t)\right)$, which is parametrized in $\mu_{\mathrm{RR}}\left(t, \mathcal{H}_{t}^{a}, \xi^{a}(t)\right)$ and $\xi_{0}^{a}(t)$, and $f^{b}\left(t \mid \mathcal{H}_{t}^{b}, \xi^{b}(t)\right)$, which is parametrized in $\mu_{\mathrm{RP}-\mathrm{RR}}\left(t, \mathcal{H}_{t}^{b}, \xi^{b}(t)\right)$ and $\xi_{0}^{b}(t)$.

The $i p \operatorname{In} f \operatorname{Tr}(t)$ definition is thus concerned with the maximum vertical distance between the cumulative distribution function of the Inverse-Gaussian distribution $F^{a}\left(t \mid \mathcal{H}_{t}^{a}, \xi^{a}(t)\right)$, which is related to the past heartbeat events exclusively, and the cumulative distribution function of the Inverse-Gaussian distribution $F^{b}\left(t \mid \mathcal{H}_{t}^{b}, \xi^{b}(t)\right)$, which is related to the past heartbeat and respiratory events. In this study, ipInfTr $(t)$ estimates were obtained setting an arbitrary value of $k=3$.

In other words, the $i p \operatorname{In} f \operatorname{Tr}(t)$ computation embeds a measure of the KS distance between two Inverse-Gaussian distributions whose first-order moments are parametrized as a monovariate and bivariate autoregressive functions, respectively.

Note that the use of an Inverse-Gaussian distribution is justified by physiological and computational reasons. In fact, Inverse-Gaussian functions are associated with an integrateand-fire model of cardiac contraction [42], and with better goodness-of-fit [42]. Since this function is formally defined at each moment in time, it is possible to obtain an instantaneous estimate of $\mu_{\mathrm{RR}}\left(t, \mathcal{H}_{t}^{a}, \xi^{a}(t)\right)$ and $\mu_{\mathrm{RP}-\mathrm{RR}}\left(t, \mathcal{H}_{t}^{b}, \xi^{b}(t)\right)$ at a very fine timescale (with an arbitrarily small bin size $\Delta$ ), requiring no interpolation between the arrival times of two beats. 


\section{E. Other Instantaneous Heartbeat Dynamics Measures}

For the sake of conciseness, we here reference to our previous publications for the description of other instantaneous heartbeat dynamics measures than ipTE and ipInfTr. In the general sense, in fact, our framework allows for a quantitative characterization of many heartbeat dynamics based on instantaneous time-, and frequency domain estimations [42], as well as complex (e.g., ipApEn [9]) and multivariate (e.g., RSA [45]) measures. Specifically, the time-domain characterization is based on the first and the second order moments of the underlying probability structure. Namely, given the time-varying parameter set $\xi(t)$, the instantaneous estimates of mean $\mu_{\mathrm{RR}}\left(t, \mathcal{H}_{t}, \xi(t)\right)$, R-R interval standard deviation $\sigma_{R R}^{2}\left(t, \mathcal{H}_{t}, \xi(t)\right)$, and heart rate standard deviation $\sigma_{H R}\left(t, \mathcal{H}_{t}, \xi(t)\right)$ can be derived at each moment in time as follows [42], [43].

The linear power spectrum estimation reveals the linear mechanisms governing the heartbeat dynamics in the frequency domain. In particular, given the model of $\mu_{\mathrm{RR}}\left(t, \mathcal{H}_{t}, \xi(t)\right)$, we can compute the time-varying parametric (linear) autospectrum [42], [43]. By integrating this autospectrum in each frequency band, we compute the indices within the low frequency $(\mathrm{LF}=0.04-0.15 \mathrm{~Hz}$ ), and high frequency $(\mathrm{HF}=0.14-0.45 \mathrm{~Hz})$ ranges, along with their ratio $(\mathrm{LF} / \mathrm{HF})$.

The instantaneous monovariate heartbeat complexity estimation, ipApEn, just like the hereby proposed $i p T E$ and ipInfTr, relies on the distance calculation of heartbeatrelated PDFs in the phase space [9]. Of note, ipApEn modeling is based on the Laguerre expansion of a nonlinear WienerVolterra representation of complex heartbeat dynamics. Finally, it is worthwhile mentioning that estimation of RSA [45] is derived from the transfer function between $\phi_{1}$ and $\phi_{2}$ of eq. 4.

\section{EXPERIMENTAL SETUP}

In this Section, we report on mathematical details of the model generating synthetic cardio-respiratory data, as well as on the experimental protocol involving healthy subjects undergoing postural changes.

\section{A. Synthetic Data}

In this study, synthetic data refers to the output of a recently proposed model of cardio-respiratory dynamics, through which it is possible to compute the exact theoretical values of standard TE measures for the simulated dynamics [46].

Briefly, the model is based on vector autoregressive processes devised to reproduce realistic cardiorespiratory interactions:

$$
\begin{aligned}
& R P_{n}=a_{1} R P_{n-1}+a_{2} R P_{n-2}+\epsilon_{n} \\
& R R_{n}=\sum_{k=1}^{4} b_{k} R R_{n-k}+c\left(R P_{n-1}-R P_{n-2}\right)+\zeta_{n}
\end{aligned}
$$

where the processes RP and RR represent the respiration and heartbeat dynamics, respectively. The terms $\epsilon_{n}$ and $\zeta_{n}$ are independent Gaussian white noises with variances set to 2 and 1 , respectively. Oscillations in the two processes at the typical frequencies of cardiorespiratory variability are ensured by placing pairs of complex-conjugated poles of magnitude $\rho$ and phase $2 \pi f$ in the complex plane representation of the processes. In particular, for the $R R$ process, we initially set very low frequency (VLF) oscillations with $\rho_{V L F}=0.2$, $f_{V L F}=0.03$, and low frequency (LF) oscillations with $\rho_{L F}=0.8, f_{L F}=0.1$, whereas for the $R P$ process we initially set $\rho_{H F}=0.9$ and $f_{H F}=0.3$.

We investigated standard TE and the proposed $i p T E$ and ipInfTr measures by varying the simulation parameters according to the following conditions:

- the coupling $c$ was changed from 0 to 1 to simulate an increasing RSA. This setting causes an increase of the HF power in the spectral density of the simulated RR interval series.

- the pole $\rho_{L F}$ was changed from 0 to 0.8 , with coupling $c=0.8-\rho_{L F}$, to simulate a shift in the sympathovagal balance toward sympathetic activation and vagal deactivation. This setting causes an increase of the LF power, and a simultaneous decrease in the HF power, in the spectral density of the simulated RR interval series

Further details on the resulting model coefficients, and the theoretical calculation of standard TE measures can be found in [46].

In order to demonstrate that proposed $i p T E$ and $i p I n f T r$ measures are able to identify the directionality of systems coupling, we also gathered estimates from the following system:

$$
\begin{array}{r}
R P_{n}=a_{1} R P_{n-1}+a_{2} R P_{n-2}+c_{r}\left(R R_{n-1}-R R_{n-2}\right)+\epsilon_{n} \\
R R_{n}=\sum_{k=1}^{4} b_{k} R R_{n-k}+\zeta_{n}
\end{array}
$$

in which the information transfer is from RR to RP through the coupling coefficient $c_{r}$ from 0 to 1 .

\section{B. Experimental Data}

To show the applicability of $i p T E$ and $i p I n f T r$ measures in actual heartbeat data, we fitted the monovariate and bivariate point-process models (see eqs. 3 and 4, respectively) using RR interval series gathered from 16 healthy subjects (10 males, range: $24-34 \mathrm{yr} ; 28.6 \pm 2.9 \mathrm{yr}$, no known history of cardiovascular disease) undergoing a tilt-table protocol. Each subject, initially lying horizontally in a supine position, is then passively tilted to the vertical position according to the following protocol: $4 \mathrm{~min}$ in early supine position, $5 \mathrm{~min}$ tilted head-up to an angle of $70^{\circ}$ and 4 min back to later supine position. Transitions from supine-to-upright and from uprightto-supine lasted about 20s each. Throughout the experiment, a 12-lead ECG was recorded using a Biopac MP150 system, with a sampling frequency of $1000 \mathrm{~Hz}$. Respiratory signal was recorded with a sampling frequency of $125 \mathrm{~Hz}$, by using TSD201 transducer which measures thoracic expansion while breathing, giving a measure correlated with lung volume changes. Arterial pressure was measured at the finger with a non-invasive device (Finometer, inopress Medical System). Further details can be found in [47], [48]. 

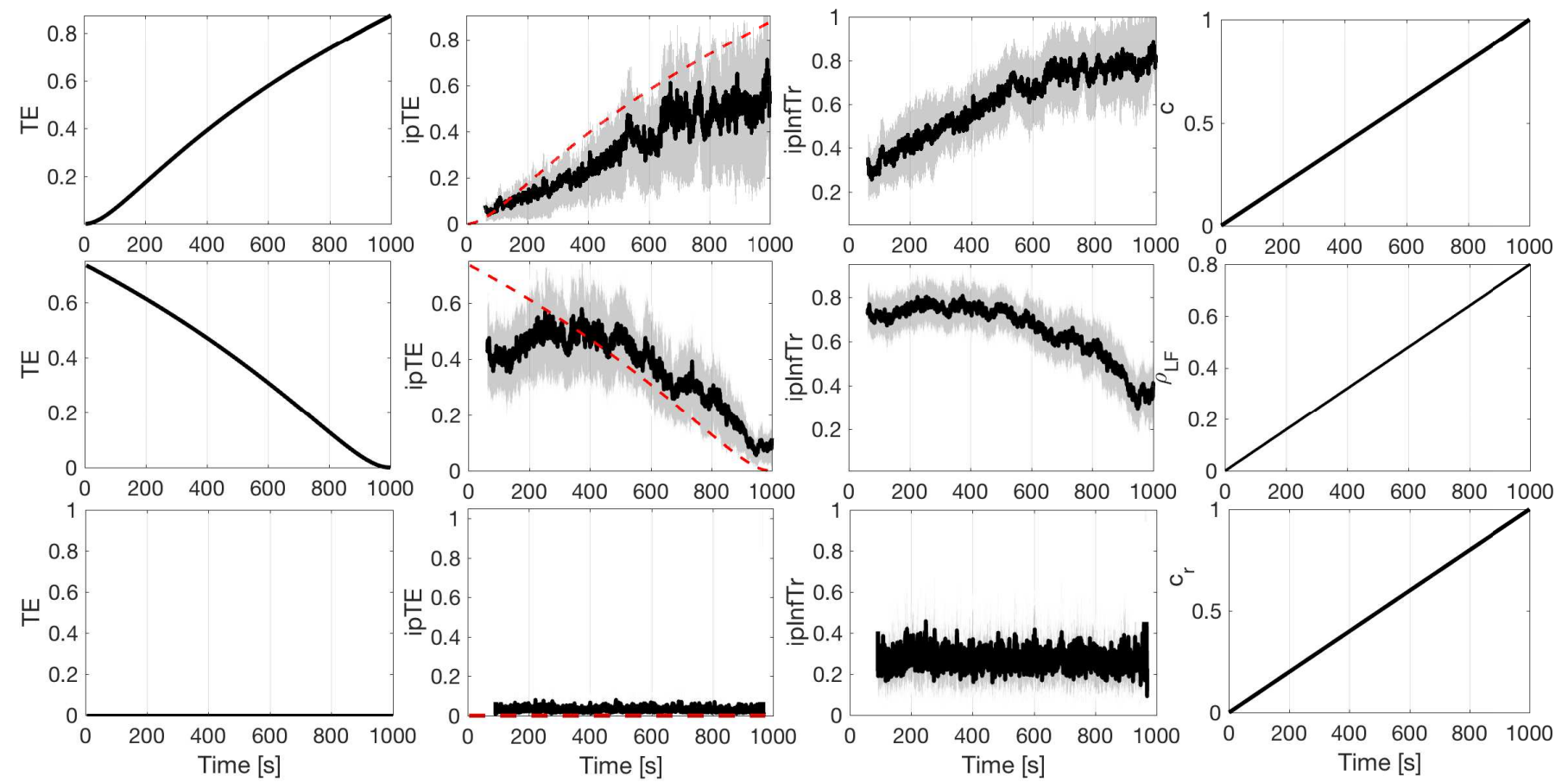

Fig. 1. Simulation results using the synthetic models of cardiorespiratory dynamics. Top row panels show results associated with change in the cardiorespiratory coupling $c=[0,1]$ (eq. 9), middle row panels show results associated with change in the pole $\rho_{L F}=[0,0.8]$ (eq. 9), whereas bottom row panels show results associated with change in the cardiorespiratory coupling $c_{r}=[0,1]$ (eq. 10). From the left, the theoretical TE, the proposed $i p T E$ and $i p I n f T r$, and the model parameter changes as a function of the time are shown. Plots of $i p T E$ and $i p I n f T r$ show instantaneous group-wise statistics expressed as $\operatorname{Median}(X)$ (black lines) Median $(|X-\operatorname{Median}(X)|)$ (grey area), calculated using 100 simulations. Theoretical values of TE are superimposed in the $i p T E$ plots (red dashed lines).

\section{RESUlts}

Given a generic index variable $X$, group-wise results are expressed as $\operatorname{Median}(X) \pm \operatorname{Median}(|X-\operatorname{Median}(X)|)$. Results obtained by processing synthetic cardiorespiratory data, as well as real heartbeat data follow below.

\section{A. Synthetic Data}

We obtained $i p T E$ and $i p I n f T r$ estimations by fitting the monovariate (eq. 3) and bivariate (eq. 4) point-process models on the synthetic $R R$ and $R P$ series derived from eq. 9 and 10. For the model in eq. 9 , series of length 1000 seconds were generated 100 times for each of the two considered conditions: $c=[0,1]$, and $\rho_{L F}=[0,0.8]$, whereas for the model in eq. 10 , series of length 1000 seconds were generated 100 times for $c_{r}=[0,1]$. The model orders were set as $p=7, q=2$ according to a preliminary KS plots goodness-of-fit analysis [42]. The resulted ipTE and ipInfTr series along with the respective theoretical TE are shown in Fig. 1, and summarized in Table I.

For $c=[0,1]$, theoretical $T E$ and $i p T E$, as well as $i p I n f T r$ increase according to $c$. Statistically, during the last 500 s of simulation, all of these measures significantly increased with respect to the ones in the first 500s $\left(p<3 * 10^{-17}\right.$ from non-parametric Wilcoxon test for paired data with null hypothesis of equal medians). For $\rho_{L F}=[0,0.8], T E$ and $i p T E$, as well as ipInfTr decrease with respect to the increase of $\rho_{L F}$. Particularly, $i p T E$ and $i p I n f T r$ decrease starts from circa 400 s, i.e., $\rho_{L F}$ greater than 0.2 . In fact, during the last 500s of simulation, all measures significantly decreased with respect to the ones in the first 500s $\left(p<2 * 10^{-17}\right.$ from non-parametric Wilcoxon test for paired data with null hypothesis of equal medians). For $c_{r}=[0,1]$, theoretical $T E$ is null, with stationary $i p T E$ values of $0.0317 \pm 0.0038$, and ipInfTr of $0.2692 \pm 0.0173$.

The proposed $i p T E$ and $i p I n f T r$ are therefore able to track the complex directional information transfer of the simulated physiological systems at each moment in time, being in agreement with theoretical TE estimates.

TABLE I

RESULTS FROM THE SYNTHETIC DATASET.

\begin{tabular}{|c|c|c|c|c|}
\hline \multirow{2}{*}{$\begin{array}{l}\text { Modulation } \\
\text { Parameter }\end{array}$} & \multirow{2}{*}{ Index } & \multicolumn{2}{|c|}{ Time [s] } & \multirow{2}{*}{ p-value } \\
\hline & & {$[0-500)$} & [500-1000] & \\
\hline \multirow{3}{*}{ c } & $T E$ & 0.2330 & 0.6997 & - \\
\hline & ipTE & $0.1564 \pm 0.0512$ & $0.4472 \pm 0.1001$ & $1.9417 \mathrm{e}-17$ \\
\hline & ipInfTr & $0.4657 \pm 0.0680$ & $0.7447 \pm 0.0633$ & $2.645 \mathrm{e}-17$ \\
\hline \multirow{3}{*}{$\rho_{L F}$} & $T E$ & 0.5777 & 0.1741 & - \\
\hline & $i p T E$ & $0.4712 \pm 0.0638$ & $0.2774 \pm 0.0554$ & $5.943 \mathrm{e}-18$ \\
\hline & ipInfTr & $0.7435 \pm 0.0359$ & $0.5864 \pm 0.0459$ & $1.373 \mathrm{e}-17$ \\
\hline
\end{tabular}

hypothesis of equal medians

\section{B. Experimental Data}

In 12 out of 16 recordings, KS plots and autocorrelation samples fell within $95 \%$ confidence intervals, whereas in the remaining $4 \mathrm{KS}$ plots were slightly outside the boundaries. 
KS distance analysis reveals a very satisfactory goodnessof-fit, being as low as $0.0390 \pm 0.0078$. According to KS analysis, we selected a order of $p=7$, and $q=2$. Results from univariate, non-parametric statistical analysis of instantaneous features related to linear and nonlinear heartbeat dynamics are summarized in Table II. Noticeably, trends in total RR mean and variability, $\mathrm{HF}$ and $\mathrm{LF} / \mathrm{HF}$ ratio, as well as in RSA are in agreement with the current knowledge associated with supine to upright changes (i.e., a reduced vagal activity and RSA is associated with upright position). Instantaneous statistics averaged among all subjects are shown in Fig. 2. Concerning the instantaneous complexity-related measures, results show a significant decrease in the inhomogeneous pointprocess approximate entropy, $i p A p E n$, confirming previous results demonstrating that upright position is associated with a decreased heartbeat complexity (see also Fig. 2). Therefore, by comparing $i p T E$ and $i p I n f T r$ with its purely monovariate counterpart $i p A p E n$, we showed similarities in the dynamics of the supine phases, and differences throughout the upright phase. A plateau, in fact, is shown by $i p T E$ and $i p I n f T r$ dynamics (see Fig. 3) despite the higher variability of $i p A p E n$ measures (see Fig. 2).

Instantaneous $i p T E_{R P \rightarrow R R}$ and $i p \operatorname{Inf} T r_{R P \rightarrow R R}$ statistics averaged among all subjects are shown in Fig. 3, whereas instantaneous $i p T E_{B P \rightarrow R R}$ and $i p \operatorname{Inf} T r_{B P \rightarrow R R}$ statistics averaged among all subjects are shown in Fig. 4. As expected, $i p$ Transf $E n_{R P \rightarrow R R}$ and and $i p I n f T r_{R P \rightarrow R R}$ significantly decreases in upright conditions, whereas $i p T E_{B P \rightarrow R R}$ and $i p \operatorname{Inf} \operatorname{Tr}_{B P \rightarrow R R}$ increase. However, $i p T E_{B P \rightarrow R R}$ and ipInf $\operatorname{Tr}_{B P \rightarrow R R}$ trends do not reach statistical significance due to a high inter-subject variability, although it is possible to appreciate a clear variable increase for around 60s following postural changes (see Fig. 4).

TABLE II

RESULTS FROM THE POSTURAL CHANGES DATASET.

\begin{tabular}{|c|c|c|c|}
\hline $\begin{array}{c}\text { Autonomic } \\
\text { Index }\end{array}$ & Supine & Upright & p-value \\
\hline \hline$\mu_{R R}[\mathrm{~ms}]$ & $984.11 \pm 57.66$ & $772.51 \pm 97.29$ & $4.4 \mathrm{e}-4$ \\
\hline$\sigma_{R R}^{2}\left[\mathrm{~ms}^{2}\right]$ & $829.78 \pm 461.84$ & $293.93 \pm 233.55$ & $9.73 \mathrm{e}-3$ \\
\hline$\sigma_{H R}^{2}\left[\right.$ beat $\left.^{2} / \mathrm{min}^{2}\right]$ & $3.57 \pm 2.04$ & $2.80 \pm 2.05$ & 0.379 \\
\hline $\mathrm{LF}\left[\mathrm{ms}^{2}\right]$ & $1233.80 \pm 677.83$ & $966.15 \pm 470.70$ & 0.379 \\
\hline $\mathrm{HF}\left[\mathrm{ms}^{2}\right]$ & $544.57 \pm 161.12$ & $185.37 \pm 148.90$ & 0.017 \\
\hline $\mathrm{LF} / \mathrm{HF}$ & $2.00 \pm 1.09$ & $11.17 \pm 7.92$ & 0.001 \\
\hline $\mathrm{ipApEn}$ & $0.340 \pm 0.042$ & $0.271 \pm 0.042$ & 0.015 \\
\hline $\mathrm{RSA}$ & $0.064 \pm 0.025$ & $0.020 \pm 0.012$ & 0.003 \\
\hline$i p T E_{R P \rightarrow R R}$ & $0.721 \pm 0.520$ & $0.097 \pm 0.056$ & 0.001 \\
\hline$i p T E_{B P \rightarrow R R}$ & $0.0082 \pm 0.0064$ & $0.0089 \pm 0.0045$ & 0.163 \\
\hline$i p I n f T r_{R P \rightarrow R R}$ & $0.649 \pm 0.207$ & $0.308 \pm 0.138$ & 0.001 \\
\hline$i p I n f T r_{B P \rightarrow R R}$ & $0.130 \pm 0.079$ & $0.118 \pm 0.041$ & 0.163 \\
\hline p-values from non-parametric Wicoxon test for paired data with nul \\
hypothesis of equal medians
\end{tabular}

\section{Discussion AND CONCLUSION}

Inspired by the standard, theoretical definition of transfer entropy (TE), we propose two novel measures of information transfer: the instantaneous point-process transfer entropy $(i p T E)$, and the instantaneous point-process Information Transfer (ipInfTr). Remarkably, these measures are able to

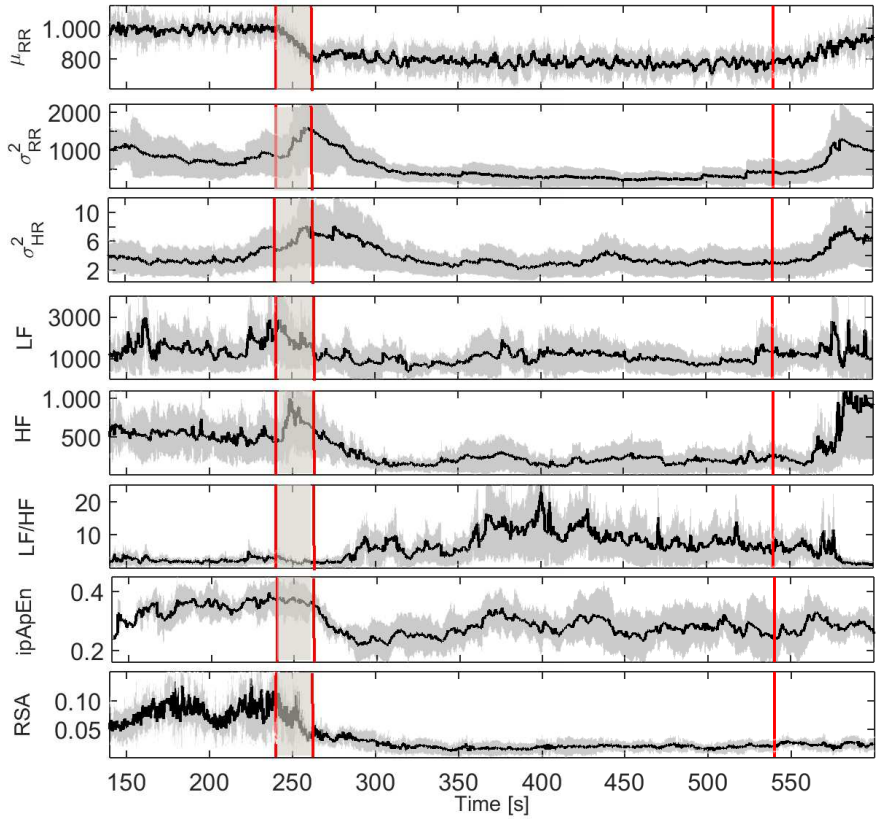

Fig. 2. Averaged instantaneous point-process statistics during resting and upright conditions. Given a generic variable $X$, considering data from all subjects, black lines indicate $\operatorname{Median}(X)$, whereas the grey area indicates Median $(\mid X-$ Median $(X) \mid))$. Vertical red lines indicate, from left to right, the end of supine condition, the beginning of the upright condition, and the end of the upright condition.

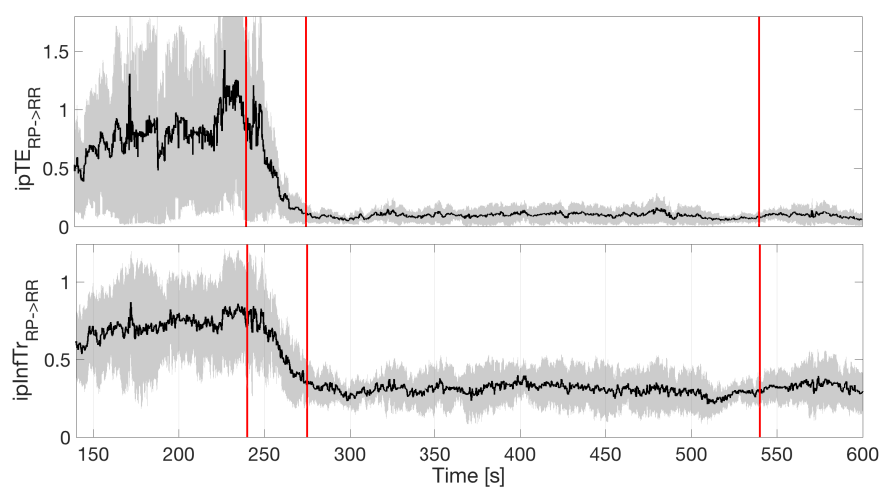

Fig. 3. Averaged $i p T E_{R P \rightarrow R R}$ (top panel) and $i p I n f T r_{R P \rightarrow R R}$ (bottom panel) group-wise statistics during resting and upright conditions. Considering data from all subjects, black lines indicate $\operatorname{Median}(X)$, whereas the grey area indicates Median $(|X-\operatorname{Median}(X)|))$. Vertical red lines indicate, from left to right, the end of supine condition, the beginning of the upright condition, and the end of the upright condition.

provide estimates of information transfer between two dynamical systems with a high-resolution in time, therefore tracking two physiological systems in non-stationary conditions. The mathematical definition, embedded into the point-process framework, ensures continuous estimates in time without the use of any interpolation procedure.

The rationale behind $i p T E$ and $i p I n f T r$ definitions relies on the non-parametric estimation of TE as a nonlinear extension of Granger causality. Leveraging on the point-process theory for cardiovascular dynamics, which associates an InverseGaussian PDF to each heartbeat events, we aimed at quantifying the distances between two distributions parametrized 


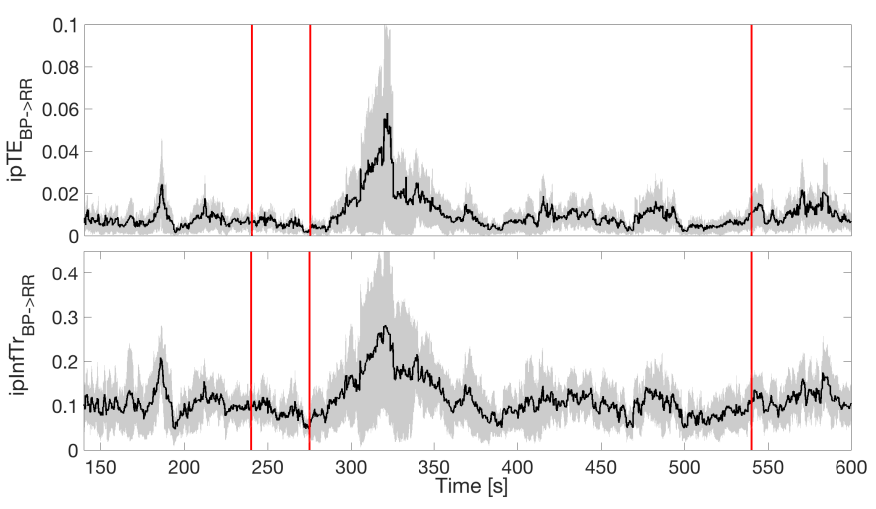

Fig. 4. Averaged $i p T E_{B P \rightarrow R R}$ (top panel) and $i p I n f \operatorname{Tr}_{B P \rightarrow R R}$ (bottom panel) group-wise statistics during resting and upright conditions. Considering data from all subjects, black lines indicate $\operatorname{Median}(X)$, whereas the grey area indicates Median $(\mid X-$ Median $(X) \mid))$. Vertical red lines indicate, from left to right, the end of supine condition, the beginning of the upright condition, and the end of the upright condition.

with the past heartbeat events (monovariate model), and the past heartbeat and respiratory/blood pressure events (bivariate model). While $i p T E$ derives from the direction application of TE over the estimated Inverse-Gaussian PDFs, ipInfTr is derived from Kolmogorov-Smirnov distance calculations of PDFs from monovariate and bivariate models. It should be noted that a shifting window approach to estimate Granger causality would only allow "discrete" estimates in time that would work exclusively at the time scale of the observations. In addition, the limited number of observations within the windowed data need to be compensated by an appropriate model in order to predict the nonlinear dynamics (and their evolution) with sufficient accuracy. To this extent, our approach combines causality with a proper mathematical framework ensuring that the estimates can actually be derived in the "continuous" time $t$, as reported in the PDF formulation, thus increasing the number of observation points to a wider range of time scales, and also defines a powerful underlying model coupled with a clear goodness-of-fit assessment that allows to test the most appropriate structures $f\left(t \mid \mathcal{H}_{t^{\prime}}, \xi\left(t^{\prime}\right)\right)$ for $t>u_{j}$.

For these reasons, the proposed indices could provide a more meaningful quantification than traditional directional entropy measures. Moreover, all other advantages of the pointprocess framework, e.g., goodness-of-fit measures such as KS distance and autocorrelation plots that quantitatively allow to verify the model fit and to choose the proper model order, are embedded in the $i p T E$ and $i p I n f T r$ definitions.

Validation on synthetic, physiologically plausible cardiorespiratory data confirmed that the proposed ipTE and ipInfTr are able to track the theoretical TE changes with a highresolution in time. Note that the constant bias shown in Fig. 1 for $i p I n f T r$ values in case of uncoupled systems is due to the specific choice of $k$ in eq. 8. Nonetheless, we demonstrated how to finely track changes in the directional cardiorespiratory coupling, as well as changes in the sympathovagal balance.

Once validated, we investigated $i p T E$ and $i p I n f T r$ dynamics in actual heartbeat data gathered from healthy subjects undergoing postural changes. By grand-averaging along the time, our experimental results are consistent with previous experi- mental TE estimates during postural changes [30], [31], [34][38]. Estimates of $i p T E_{R P \rightarrow R R}$ and $i p T E_{B P \rightarrow R R}$ clearly reveal trends associated with decreasing cardiorespiratory information transfer and increasing cardiovascular information transfer during the transition from supine to upright position. These trends are in agreement with those observed previously using standard linear and nonlinear measures of TE and Granger causality [30], [31], [36], [49]. In addition, the high temporal resolution of the proposed estimates allowed us to track specific trends, such as those related to the prompt response to tilt of $i p T E_{R P \rightarrow R R}$ and $i p \operatorname{Inf} T r_{R P \rightarrow R R}$, which decrease rapidly and are kept at low values throughout the test (Fig. 3), or the different response of $i p T E_{B P \rightarrow R R}$ and ipInf $\operatorname{Tr}_{B P \rightarrow R R}$, which raise with a certain latency and is not stable throughout the test (Fig. 4). Differently from $i p T E_{R P \rightarrow R R}$ and ipInfTr $\operatorname{Tr}_{R P \rightarrow R R}$ trends, we found that $i p T E_{B P \rightarrow R R}$ and $i p I n f \operatorname{Tr}_{B P \rightarrow R R}$ estimates are associated with a high inter-subject variability. Particularly, during the upright phase, $i p T E_{B P \rightarrow R R}$ and $i p \operatorname{Inf} \operatorname{Tr}_{B P \rightarrow R R}$ reach a maximal value after about 1 minute from tilting. However, both $i p T E_{B P \rightarrow R R}$ and $i p I n f T r_{B P \rightarrow R R}$ start increasing after about 30s. These dynamics are consistent with the fact that each subject transitioned from supine to the upright position in about 20s, and that the characteristic autonomic response generates oscillations at around $0.1 \mathrm{~Hz}$, i.e., $10 \mathrm{~s}$.

From a physiological point of view, we have shown that $i p T E$ and $i p I n f T r$ promisingly provides helpful multivariate time-varying and adaptive assessment for real-time monitoring of sympathovagal dynamics, which have also been proven in agreement with previous works [50]. Furthermore, $i p T E_{R P \rightarrow R R}$ and $i p I n f T r_{R P \rightarrow R R}$ are here applied to cardiorespiratory dynamics, and it can consequently be linked to respiratory sinus arrhythmia (RSA). It is also known that RSA interacts with the baroreflex, as confirmed by previous studies highlighting the causal relation between them [51]. As a matter of fact, this study indeed shows that $i p T E_{B P \rightarrow R R}$ and ipInf $\operatorname{Tr}_{B P \rightarrow R R}$ dynamics follow a similar behaviour as it has been observed for baroreflex sensitivity [24]. We observed different dynamics on group-wise statistics of $i p T E$ and ipInfTr between the supine-to-upright phase and uprightto-supine phase. Differences between these two phases have already been highlighted in the literature (see, e.g., [24], [52][55]). Consistently with this literature, sympathetic withdrawal and the restoring of resting-state vagal activity levels during the upright-to-supine transition seem to occur with delayed, slower dynamics, clearly different than the supine-to-upright phase. At a speculative level, the slower vagal reactivation, or even more probably, the slower sympathetic withdrawal during recovery might be due to a delayed re-synchronization at a central (brainstem) level, as also highlighted in parallel mechanisms investigated in post-exercise recovery [56]. Nevertheless, the detailed physiological mechanisms are still unknown, and further investigation would entail inclusion of the time-varying behaviour of cardiovascular variables beyond heart rate variability.

We have also proved that our novel ipTE and ipInfTr measures are able to overcome some of the inter-individual variability shown by a monovariate complex HRV assessment 
(e.g., $i p A p E n)$

Similarly to the recently proposed complexity variability framework [8], the proposed entropy measures also allow for the study of multivariate complexity variability, i.e., the analysis of coupled interacting complex systems, referring to the fluctuations in multivariate complexity instead of analysis of central tendency exclusively. We remark that the proposed methodology has been derived to quantify the statistical coherence between nonlinear systems evolving in time. Nevertheless, from a theoretical (and philosophical) perspective its not straightforward to discern behaviours of physiological nonlinearity from non-stationarity [57]. It could be possible, in fact, to consider simple, possibly multivariate, linear models with non-stationary transition dynamics [58], or a single nonlinear model with multiple operating regimes [59]. Our approach concerns multivariate, non-stationarity physiological systems as modelled through multivariate linear equations, therefore complying with non-stationarity, linear physiological systems, or nonlinear physiological systems whose nonlinearity is derived from non-stationarity. Indeed, a single nonlinear model with multiple operating regimes could be approximated with a linear non-stationary model. Moreover, our use of linear parametric models to predict non-Gaussian (i.e., Inverse-Gaussian) statistics should capture some of the cardiovascular system nonlinearity. This is different from the approach proposed in most of our previous studies (e.g., [8], [9], [17], [60][62]) which dealt with monovariate nonlinear, non-stationary physiological systems.

To conclude, the proposed methodology offers a promising mathematical tool for the dynamic analysis of a wide range of applications and to potentially study any physical and natural stochastic discrete process (e.g. [43]). We envisage significant avenues in the study of hidden, transient, non-stationary physiological states involving multivariate autonomic dynamics in health and disease. Furthermore, the flexible definition of $i p T E$ and ipInfTr, which is not limited to bivariate formulations or strictly linked to specific physiological systems, allows for future tailoring of the model to the definition of fully multivariate instantaneous measures of information transfer and to the study of complex multi-system physiology such as brain-heart interactions or, more in general, brain-body interactions.

\section{REFERENCES}

[1] G. Captur, A. L. Karperien, A. D. Hughes, D. P. Francis, and J. C. Moon, "The fractal heart [mdash] embracing mathematics in the cardiology clinic," Nature Reviews Cardiology, 2016.

[2] L. Glass, "Dynamical disease: Challenges for nonlinear dynamics and medicine," Chaos: An Interdisciplinary Journal of Nonlinear Science, vol. 25, no. 9, p. 097603, 2015.

[3] U. Rajendra Acharya, K. Paul Joseph, N. Kannathal, C. Lim, and J. Suri, "Heart rate variability: a review," Medical and Biological Engineering and Computing, vol. 44, no. 12, pp. 1031-1051, 2006.

[4] A. Goldberger, C. Peng, and L. Lipsitz, "What is physiologic complexity and how does it change with aging and disease?" Neurobiology of aging, vol. 23, no. 1, pp. 23-26, 2002.

[5] R. Sassi, S. Cerutti, F. Lombardi, M. Malik, H. V. Huikuri, C.-K. Peng, G. Schmidt, Y. Yamamoto, B. Gorenek, G. H. Lip et al., "Advances in heart rate variability signal analysis: joint position statement by the ecardiology esc working group and the european heart rhythm association co-endorsed by the asia pacific heart rhythm society," Europace, p. euv015, 2015.
[6] A. Voss, S. Schulz, R. Schroeder, M. Baumert, and P. Caminal, "Methods derived from nonlinear dynamics for analysing heart rate variability," Philosophical Transactions of the Royal Society A: Mathematical, Physical and Engineering Sciences, vol. 367, no. 1887, pp. 277-296, 2009.

[7] A. Armoundas, K. Ju, N. Iyengar, J. Kanters, P. Saul, and others., "A stochastic nonlinear autoregressive algorithm reflects nonlinear dynamics of heart-rate fluctuations," Annals of biomedical engineering, vol. 30, no. 2, pp. 192-201, 2002.

[8] G. Valenza, L. Citi, and R. Barbieri, "Estimation of instantaneous complex dynamics through lyapunov exponents: a study on heartbeat dynamics," PloS one, vol. 9, no. 8, p. e105622, 2014.

[9] G. Valenza, L. Citi, E. P. Scilingo, and R. Barbieri, "Inhomogeneous point-process entropy: An instantaneous measure of complexity in discrete systems," Physical Review E, vol. 89, no. 5, p. 052803, 2014.

[10] H. Joe, "Relative entropy measures of multivariate dependence," Journal of the American Statistical Association, vol. 84, no. 405, pp. 157-164, 1989.

[11] Karmeshu, Entropy measures, maximum entropy principle and emerging applications. Springer, 2003, vol. 119.

[12] A. Porta, F. Aletti, F. Vallais, and G. Baselli, "Multimodal signal processing for the analysis of cardiovascular variability," Philosophical Transactions of the Royal Society A, vol. 367, no. 1887, pp. 391-409, 2009.

[13] K. Sunagawa, T. Kawada, and T. Nakahara, "Dynamic nonlinear vagosympathetic interaction in regulating heart rate," Heart and Vessels, vol. 13, no. 4, pp. 157-174, 1998.

[14] P. C. Ivanov, L. N. Amaral, A. L. Goldberger, and H. E. Stanley, "Stochastic feedback and the regulation of biological rhythms," EPL (Europhysics Letters), vol. 43, no. 4, p. 363, 1998.

[15] C. Schäfer, M. G. Rosenblum, J. Kurths, and H.-H. Abel, "Heartbeat synchronized with ventilation," Nature, vol. 392, pp. 239-240, 1998.

[16] "Special issues on nonlinearity on heart rate," Chaos, vol. 19, 2009.

[17] G. Valenza, L. Citi, A. Lanatá, E. P. Scilingo, and R. Barbieri, "Revealing real-time emotional responses: a personalized assessment based on heartbeat dynamics," Scientific reports, vol. 4, 2014.

[18] C. Poon and C. Merrill, "Decrease of cardiac chaos in congestive heart failure," Nature, vol. 389, no. 6650, pp. 492-495, 1997.

[19] G. Valenza, A. Lanata, and E. P. Scilingo, "Oscillations of heart rate and respiration synchronize during affective visual stimulation," Information Technology in Biomedicine, IEEE Transactions on, vol. 16, no. 4, pp. 683-690, 2012.

[20] A. Winfree, "Electrical turbulence in three-dimensional heart muscle," Science, vol. 266, no. 5187, pp. 1003-1006, 1994.

[21] G. Valenza, M. Nardelli, A. Lanata, C. Gentili, G. Bertschy, R. Paradiso, and E. P. Scilingo, "Wearable monitoring for mood recognition in bipolar disorder based on history-dependent long-term heart rate variability analysis," Biomedical and Health Informatics, IEEE Journal of, 2014.

[22] D. R. Chialvo, R. F. Gilmour Jr, and J. Jalife, "Low dimensional chaos in cardiac tissue," Nature, vol. 343, no. 6259, pp. 653-657, 1990.

[23] M. P. Tarvainen, S. Georgiadis, T. Laitio, J. A. Lipponen, P. A. Karjalainen, K. Kaskinoro, and H. Scheinin, "Heart rate variability dynamics during low-dose propofol and dexmedetomidine anesthesia," Annals of biomedical engineering, vol. 40, no. 8, pp. 1802-1813, 2012.

[24] M. Orini, P. Laguna, L. Mainardi, and R. Bailón, "Assessment of the dynamic interactions between heart rate and arterial pressure by the cross time-frequency analysis," Physiological measurement, vol. 33, no. 3, p. 315,2012

[25] G. Valenza, M. Nardelli, G. Bertschy, A. Lanata, and E. Scilingo, "Mood states modulate complexity in heartbeat dynamics: A multiscale entropy analysis," EPL (Europhysics Letters), vol. 107, no. 1, p. 18003, 2014.

[26] P. Schwartz and G. De Ferrari, "Sympathetic-parasympathetic interaction in health and disease: abnormalities and relevance in heart failure," Heart failure reviews, pp. 1-7, 2010.

[27] S. Akselrod, D. Gordon, F. A. Ubel, D. C. Shannon, A. Berger, R. J. Cohen et al., "Power spectrum analysis of heart rate fluctuation: a quantitative probe of beat-to-beat cardiovascular control," science, vol. 213, no. 4504, pp. 220-222, 1981.

[28] F. Yasuma and J.-i. Hayano, "Respiratory sinus arrhythmia: why does the heartbeat synchronize with respiratory rhythm?" Chest Journal, vol. 125, no. 2, pp. 683-690, 2004.

[29] K. Hlaváčková-Schindler, M. Paluš, M. Vejmelka, and J. Bhattacharya, "Causality detection based on information-theoretic approaches in time series analysis," Physics Reports, vol. 441, no. 1, pp. 1-46, 2007.

[30] L. Faes, G. Nollo, and A. Porta, "Information-based detection of nonlinear granger causality in multivariate processes via a nonuniform embedding technique," Physical Review E, vol. 83, no. 5, p. 051112, 2011. 
[31] A. Porta, A. M. Catai, A. C. Takahashi, V. Magagnin, T. Bassani, E. Tobaldini, P. Van De Borne, and N. Montano, "Causal relationships between heart period and systolic arterial pressure during graded headup tilt," American Journal of Physiology-Regulatory, Integrative and Comparative Physiology, vol. 300, no. 2, pp. R378-R386, 2011.

[32] S. Nemati, B. A. Edwards, J. Lee, B. Pittman-Polletta, J. P. Butler, and A. Malhotra, "Respiration and heart rate complexity: effects of age and gender assessed by band-limited transfer entropy," Respiratory physiology \& neurobiology, vol. 189, no. 1, pp. 27-33, 2013.

[33] L. Faes, G. Nollo, F. Jurysta, and D. Marinazzo, "Information dynamics of brain-heart physiological networks during sleep," New Journal of Physics, vol. 16, no. 10, p. 105005, 2014.

[34] M. Staniek and K. Lehnertz, "Symbolic transfer entropy: inferring directionality in biosignals," Biomedizinische Technik/Biomedical Engineering, vol. 54, no. 6, pp. 323-328, 2009.

[35] L. Faes, G. Nollo, and A. Porta, "Compensated transfer entropy as a tool for reliably estimating information transfer in physiological time series," Entropy, vol. 15, no. 1, pp. 198-219, 2013.

[36] A. Porta, L. Faes, G. Nollo, V. Bari, A. Marchi, B. De Maria, A. C. Takahashi, and A. M. Catai, "Conditional self-entropy and conditional joint transfer entropy in heart period variability during graded postural challenge," PloS one, vol. 10, no. 7, p. e0132851, 2015.

[37] M. Lungarella, A. Pitti, and Y. Kuniyoshi, "Information transfer at multiple scales," Physical Review E, vol. 76, no. 5, p. 056117, 2007.

[38] A. Porta and L. Faes, "Wiener-granger causality in network physiology with applications to cardiovascular control and neuroscience," Proceedings of the IEEE, vol. 104, no. 2, pp. 282-309, 2016.

[39] Y. Li, H.-L. Wei, S. A. Billings, and X.-F. Liao, "Time-varying linear and nonlinear parametric model for granger causality analysis," Physical Review E, vol. 85, no. 4, p. 041906, 2012.

[40] G. Valenza, L. Faes, L. Citi, M. Orini, and R. Barbieri, "Instantaneous transfer entropy for the study of cardio-respiratory dynamics," in Engineering in Medicine and Biology Society (EMBC), 2015 37th Annual International Conference of the IEEE. IEEE, 2015, pp. 7885-7888.

[41] P. Wollstadt, M. Martínez-Zarzuela, R. Vicente, F. J. Díaz-Pernas, and M. Wibral, "Efficient transfer entropy analysis of non-stationary neural time series," PloS one, vol. 9, no. 7, p. e102833, 2014.

[42] R. Barbieri, E. Matten, A. Alabi, and E. Brown, "A point-process model of human heartbeat intervals: new definitions of heart rate and heart rate variability," American Journal of Physiology-Heart and Circulatory Physiology, vol. 288, no. 1, p. H424, 2005.

[43] G. Valenza, L. Citi, E. Scilingo, and R. Barbieri, "Point-process nonlinear models with laguerre and volterra expansions: Instantaneous assessment of heartbeat dynamics," Signal Processing, IEEE Transactions On, vol. 61, no. 11, pp. 2914-2926, 2013.

[44] L. Barnett, A. B. Barrett, and A. K. Seth, "Granger causality and transfer entropy are equivalent for gaussian variables," Physical review letters, vol. 103 , no. 23 , p. 238701, 2009.

[45] Z. Chen, E. Brown, and R. Barbieri, "Assessment of autonomic control and respiratory sinus arrhythmia using point process models of human heart beat dynamics," Biomedical Engineering, IEEE Transactions on, vol. 56, no. 7, pp. 1791-1802, 2009.

[46] L. Faes, D. Widjaja, S. Van Huffel, and G. Nollo, "Investigating cardiac and respiratory determinants of heart rate variability in an informationtheoretic framework," in 2014 36th Annual International Conference of the IEEE Engineering in Medicine and Biology Society. IEEE, 2014, pp. 6020-6023.

[47] M. Orini, R. Bailón, P. Laguna, L. T. Mainardi, and R. Barbieri, "A multivariate time-frequency method to characterize the influence of respiration over heart period and arterial pressure," EURASIP Journal on Advances in Signal Processing, vol. 2012, no. 1, pp. 1-17, 2012.

[48] A. Mincholé, E. Pueyo, J. F. Rodríguez, E. Zacur, M. Doblaré, and P. Laguna, "Quantification of restitution dispersion from the dynamic changes of the $t$-wave peak to end, measured at the surface ecg," IEEE Transactions on Biomedical Engineering, vol. 58, no. 5, pp. 1172-1182, 2011.

[49] L. Faes, A. Porta, and G. Nollo, "Information decomposition in bivariate systems: theory and application to cardiorespiratory dynamics," Entropy, vol. 17, no. 1, pp. 277-303, 2015.

[50] A. Porta, T. Gnecchi-Ruscone, E. Tobaldini, S. Guzzetti, R. Furlan, and N. Montano, "Progressive decrease of heart period variability entropy-based complexity during graded head-up tilt," Journal of applied physiology, vol. 103, no. 4, pp. 1143-1149, 2007.

[51] D. L. Eckberg, "Point: counterpoint: respiratory sinus arrhythmia is due to a central mechanism vs. respiratory sinus arrhythmia is due to the baroreflex mechanism," Journal of applied physiology, vol. 106, no. 5, pp. 1740-1742, 2009
[52] A. Porta, L. Faes, A. Marchi, V. Bari, B. De Maria, S. Guzzetti, R. Colombo, and F. Raimondi, "Disentangling cardiovascular control mechanisms during head-down tilt via joint transfer entropy and selfentropy decompositions," Frontiers in physiology, vol. 6, 2015.

[53] C. Keyl, A. Schneider, M. Dambacher, and L. Bernardi, "Time delay of vagally mediated cardiac baroreflex response varies with autonomic cardiovascular control," Journal of Applied Physiology, vol. 91, no. 1, pp. 283-289, 2001.

[54] M. Elstad, K. Toska, K. H. Chon, E. A. Raeder, and R. J. Cohen, "Respiratory sinus arrhythmia: opposite effects on systolic and mean arterial pressure in supine humans," The Journal of physiology, vol. 536, no. 1, pp. 251-259, 2001.

[55] K.-I. Iwasaki, R. Zhang, J. H. Zuckerman, J. A. Pawelczyk, and B. D. Levine, "Effect of head-down-tilt bed rest and hypovolemia on dynamic regulation of heart rate and blood pressure," American Journal of Physiology-Regulatory, Integrative and Comparative Physiology, vol. 279, no. 6, pp. R2189-R2199, 2000.

[56] M. Javorka, I. Žila, T. Balhárek, and K. Javorka, "On-and off-responses of heart rate to exercise-relations to heart rate variability," Clinical physiology and functional imaging, vol. 23, no. 1, pp. 1-8, 2003.

[57] D. T. Kaplan, "Nonlinearity and nonstationarity: the use of surrogate data in interpreting fluctuations," Studies in Health Technology and Informatics, pp. 15-281, 1997.

[58] L.-w. Lehman, R. Mark, and S. Nemati, "A model-based machine learning approach to probing autonomic regulation from nonstationary vitalsigns time series," IEEE Journal of Biomedical and Health Informatics, 2017.

[59] L. Glass, "Synchronization and rhythmic processes in physiology," Nature, vol. 410, no. 6825, pp. 277-284, 2001.

[60] G. Valenza, L. Citi, C. Gentili, A. Lanatá, E. Scilingo, R. Barbieri et al., "Point-process nonlinear autonomic assessment of depressive states in bipolar patients," Methods of Information in Medicine, vol. 53, no. 4, pp. 296-302, 2014.

[61] G. Valenza, A. Greco, L. Citi, M. Bianchi, R. Barbieri, and E. Scilingo, "Inhomogeneous point-processes to instantaneously assess affective haptic perception through heartbeat dynamics information," Scientific reports, vol. 6, 2016.

[62] G. Valenza, L. Citi, R. G. Garcia, J. N. Taylor, N. Toschi, and R. Barbieri, "Complexity variability assessment of nonlinear time-varying cardiovascular control," Scientific reports, vol. 7, 2017.

\section{APPENDIX}

The inverse-Gaussian (IG) distribution with mean $\mu$ and shape factor $\xi_{0}$ has probability density:

$$
f\left(x ; \mu, \xi_{0}\right)=\left(\frac{\xi_{0}}{2 \pi x^{3}}\right)^{\frac{1}{2}} \exp \left[\frac{-\xi_{0}(x-\mu)^{2}}{2 \mu^{2} x}\right]
$$

If $X \sim I G\left(\mu, \xi_{0}\right)$, then the following relationships hold:

$$
\mathbb{E}_{I G\left(\mu, \xi_{0}\right)}[X]=\mu, \quad \mathbb{E}_{I G\left(\mu, \xi_{0}\right)}\left[\frac{1}{X}\right]=\frac{1}{\mu}+\frac{1}{\xi_{0}}
$$

The Kullback-Leibler (KL) divergence from a distribution $Q$ to a distribution $P$ is defined to be the integral:

$$
D_{\mathrm{KL}}(P \| Q)=\mathbb{E}_{p}\left[\ln \frac{p(x)}{q(x)}\right]=\int_{-\infty}^{\infty} p(x) \ln \frac{p(x)}{q(x)} \mathrm{d} x,
$$

where $p(x)$ is the density of $P$ and $q(x)$ is the density of $Q$.

For two IG distributions $I G\left(\mu, \xi_{0}\right)$ and $I G\left(\mu^{\prime}, \xi_{0}^{\prime}\right)$, the KL divergence is:

$$
\begin{aligned}
& D_{\mathrm{KL}}\left(I G\left(\mu^{\prime}, \xi_{0}^{\prime}\right) \| I G\left(\mu, \xi_{0}\right)\right)=\int_{-\infty}^{\infty} f\left(x ; \mu^{\prime}, \xi_{0}^{\prime}\right) \ln \frac{f\left(x ; \mu^{\prime}, \xi_{0}^{\prime}\right)}{f\left(x ; \mu, \xi_{0}\right)} \mathrm{d} x \\
& =\int_{-\infty}^{\infty} f\left(x ; \mu^{\prime}, \xi_{0}^{\prime}\right)\left[\frac{1}{2} \ln \frac{\xi_{0}^{\prime}}{\xi_{0}}-\frac{\xi_{0}^{\prime}\left(x-\mu^{\prime}\right)^{2}}{2 \mu^{\prime 2} x}+\frac{\xi_{0}(x-\mu)^{2}}{2 \mu^{2} x}\right] \mathrm{d} x \\
& =\frac{1}{2} \mathbb{E}_{I G\left(\mu^{\prime}, \xi_{0}^{\prime}\right)}\left[\ln \frac{\xi_{0}^{\prime}}{\xi_{0}}+\frac{2 \xi_{0}^{\prime}}{\mu^{\prime}}-\frac{2 \xi_{0}}{\mu}+\left(\frac{\xi_{0}}{\mu^{2}}-\frac{\xi_{0}^{\prime}}{\mu^{\prime 2}}\right) X+\left(\xi_{0}-\xi_{0}^{\prime}\right) \frac{1}{X}\right] \\
& =\frac{1}{2}\left[\ln \frac{\xi_{0}^{\prime}}{\xi_{0}}+\frac{2 \xi_{0}^{\prime}}{\mu^{\prime}}-\frac{2 \xi_{0}}{\mu}+\left(\frac{\xi_{0}}{\mu^{2}}-\frac{\xi_{0}^{\prime}}{\mu^{\prime 2}}\right) \mu^{\prime}+\left(\xi_{0}-\xi_{0}^{\prime}\right)\left(\frac{1}{\mu^{\prime}}+\frac{1}{\xi_{0}^{\prime}}\right)\right] \\
& =\frac{1}{2}\left[\ln \frac{\xi_{0}^{\prime}}{\xi_{0}}+\frac{\xi_{0}}{\xi_{0}^{\prime}}-1+\frac{\xi_{0}\left(\mu^{\prime}-\mu\right)^{2}}{\mu^{\prime} \mu^{2}}\right] .
\end{aligned}
$$

